\title{
Editorial: Investigación y Experiencias Educativas Innovadoras en los procesos de aprendizaje
}

\author{
Editorial: Research and innovative educational experiences in learning processes
}

\author{
William Oswaldo Flores López ${ }^{1}$ \\ Eugenio Casimiro López Mairena ${ }^{2}$
}

\section{Justificación del monográfico}

Hablar de investigación y experiencias educativas innovadoras en los procesos de aprendizaje, del sentido y las implicaciones que ello comporta, es un tema que surge de la identificación de las situaciones problemáticas reflejadas en los informes de la “Tendencia de la Educación Superior en América Latina y el Caribe" (IELSALC, 2008); la eficacia escolar y calidad educativa en Nicaragua (IEEPP, 2016), el informe sobre desarrollo mundial "aprender para hacer realidad la promesa sobre la educación" (Banco Mundial, 2018); e investigaciones sobre dificultades en los métodos de enseñanza y los aprendizaje de las matemáticas y su relación con el estudiantado y profesorado (Romero-Díaz \& Guzmán-Contreras, 2016; Flores-Morales \& Zamora-Díaz, 2016; Flores \& López-Mairena, 2016; Flores \& Olivar-Molina, 2016; Jiménez-Bonilla \& Flores, 2017; Suárez-Martínez, Martínez-Ortiz \& López-Mairena, 2017; Alemán-Gudiel, AlemánGudiel \& López-Mairena, 2017; Flores \& Auzmendi, 2018). Presentan la tendencia a mantener las desigualdades en la educación en las sociedades de la región y se sintetiza de la manera siguiente:

- La disparidad en los logros de aprendizaje y la desigualdad en el acceso a oportunidades educativas de calidad. Se destaca que "a pesar del progreso alcanzado durante los últimos años, persisten deficiencias e insuficiencias de formación en la educación en todos los niveles de la educación, aún en los niveles más avanzados, que evidencian una brecha "digital", de capacidad cognitiva y de conocimiento y competencias entre los diferentes estratos en cada país y entre las sociedades de la región" (IESALC, 2008, p.133).

- El bajo nivel de acceso a las IES que, en gran medida, obedece a que la noción de la diversidad como derecho humano individual y de grupos no ha sido reconocida en el ámbito de la educación superior. Al respecto el informe reporta la necesidad de identificar habilidades necesarias para optar y acceder a las IES y de definir y aplicar políticas de inclusión, calidad y equidad.

1 Doctor en Educación. Profesor Investigador de la Universidad de las Regiones Autónomas de la Costa Caribe Nicaragüense. Correo: William.flores@uraccan.edu.ni (1): https://orcid.org/oooo-ooo2-1016-162o

2 Doctor en Innovación en la formación. Vicerrector de la Universidad de las Regiones Autónomas de la Costa Caribe NicaragüenseRecinto Universitario de Nueva Guinea. Correo: eugenio.lopez@uraccan.edu.ni @i: https://orcid.org/oooo-ooo2-7929-7817

Recibido: 30/04/2018 Aprobado: 01/09/2018 


\section{EDITORIAL}

- La exigencia a las IES formadora de docentes de responder al desafío de capacitar a la nueva generación de docentes para incorporar en sus clases las nuevas herramientas de aprendizaje, en un ambiente proporcionado por las TIC.

- El bajo nivel de las competencias matemáticas del profesorado de educación secundaria, en el sentido de formación de conocimientos, habilidades y actitudes para que el estudiantado sea competente en la solución de los exámenes de admisión de las universidades públicas y comunitarias (Romero-Díaz \& Guzmán-Contreras, 2016).

- Los resultados de las pruebas SERCE y TERCE demuestran que el estudiantado nicaragüense tiene el nivel de desempeño en matemática entre los niveles básicos e intermedios en relación con el resto de América Latina y el Caribe (IEEPP, 2016).

- Existe una crisis de aprendizaje en relación a las competencias de matemáticas y lengua y literatura debido que los resultados de aprendizaje son poco satisfactorios: niveles bajos, desigualdad elevada y avances lentos (Banco Mundial, 2018). La crisis del aprendizaje amplía la desigualdad: perjudica gravemente a los jóvenes desfavorecidos, que son los que más necesitan el impulso que una buena educación puede proporcionar.

- La crisis de aprendizaje se produce si fallan cuatro factores inmediatos: Docentes pocos calificados y desmotivados; estudiantes pocos preparados; gestión de las escuelas que no tiene efecto en la enseñanza ni en el aprendizaje; insumos escolares que no tienen efecto en la enseñanza ni en el aprendizaje (Banco Mundial, 2018).

- Deserción del estudiantado por factores: emotivos, académicos, económicos, motivacionales, normativos, ambientes familiares, orientación profesional, absentismo por género (Femenino); marginación cultural, étnico, lingüísticos, discapacidad y procesos de acompañamiento al estudiantado (Flores et al., 2016; Flores \& Auzmendi, 2018).

En este sentido, la Comunidad Matemática de la Costa Caribe Nicaragüense, presenta el Volumen 1, Número 2, 2018 de la Revista Electrónica de Conocimientos, Saberes y Prácticas, titulado Investigación y Experiencias Educativas Innovación en los procesos de aprendizaje (Flores \& López-Mairena, 2018) con la finalidad de contribuir al mejoramiento de la práctica docente del profesorado de educación secundaria, técnica y superior, es decir, mejorar la calidad de la educación y de los aprendizajes esta idea puede resumirse en la expresión "todo por el aprendizaje" (CASER, 2017 citado por el Banco Mundial, 2018).

A como explica el Banco Mundial (2018), comprometerse todos con el aprendizaje $y$, por lo tanto, con el aprendizaje para todos, implica poner en práctica estrategia complementarias que contribuyan a fortalecer la educación: (1) Aprender más sobre el nivel de aprendizaje para que su mejor sea un objetivo formal y medible. Medir el 
aprendizaje y mejorar sus seguimientos; utilizar esos resultados para orientar las medidas que deban adoptarse; (2) Basar el diseño de políticas en la evidencia para lograr que las escuelas estén al servicio del aprendizaje de todos los estudiantes. Utilizar la evidencia para orientar la innovación y la práctica; y (3) Construir coaliciones y alinear a los actores para que todo el sistema favorezca el aprendizaje. Abordar obstáculos técnicos y políticos que impiden el aprendizaje a escala.

\section{Estructura y presentación del monográfico}

El número se ha organizado en torno a tres grandes secciones: investigaciones educativas, Innovaciones Educativas y Reflexiones Educativas, las cuales contienen 7 artículos. A continuación, se detallan:

Este monográfico empieza con la sección de investigación educativa con los autores García-Soto, Flores \& Olivar-Molina (2018) sobre las competencias emocionales en la formación del profesorado de educación secundaria y su relación con las actitudes hacia las matemáticas, donde se resalta que las competencias emocionales en la formación del profesorado de educación secundaria se relacionan con las actitudes hacia las matemáticas, porque existen un marco de referencia que se establece en el perfil de formación docente, además, que las actitudes del profesorado hacia las matemáticas son positivas, todo esto, se interpreta como una articulación en los procesos de enseñanza y aprendizaje de las matemáticas.

El siguiente artículo es una investigación de Pérez-Obregón \& Romero-Díaz (2018) que presenta una aplicación del modelo de regresión logística, el cual permitió calcular el intervalo promedio de los estudiantes, considerando grado, sexo y año; siendo este modelo un complemento del anterior, ya que fortalece los resultados para su respectivo análisis, ambos modelos resultaron significativos y superaron los supuestos estadísticos, por tanto, se recomienda usarlos para mejorar el rendimiento del centro educativo. La sección de investigación educativa finaliza con el artículo de Aragón-Arguello \& López-Mairena (2018) sobre la enseñanza y aprendizaje del área y perímetro de polígonos regulares: una propuesta didáctica y evaluación en educación primaria, los principales resultados permitieron identificar las concepciones iniciales de los estudiantes frente a las temáticas de área y perímetro en figuras planas y sus dificultades para la interpretación de su entorno desde la Geometría. Además, se abordan aspectos relacionados con el modelo pedagógico socio crítico propio del contexto de la Escuela Cristo Redentor en la cual se desarrolló el trabajo de investigación.

En la sección de innovación educativas, se presenta la innovación de Flores (2018) sobre evaluación de los aprendizajes en la educación intercultural, fomentando que una evaluación de los aprendizajes inclusiva para poblaciones en contextos multiculturales, tiene que contribuir a la reducción de situaciones de desigualdad y exclusión social, así como, promover una evaluación para todos y con todos, teniendo de referencia el 


\section{EDITORIAL}

reconocimiento de la diversidad de conocimientos y experiencias, y la incorporación de tecnologías accesibles que posibiliten un aprendizaje para toda la vida. Seguidamente, se presenta la innovación de Orozco-Alvarado \& Díaz-Pérez (2018) sobre ¿Cómo redactar los antecedentes en una investigación cualitativa?, su propósito pretende facilitar la construcción de los antecedentes de la investigación cualitativa y, por ende, ser un referente para quienes flamantemente se inician en el área de la investigación educativa. Finalizando, con la innovación de López-Mairena \& Calero-Borge sobre acompañamiento en procesos de formación a jóvenes líderes de los pueblos indígenas del Pacífico Centro-Norte de Nicaragua, se considera importante desarrollar estos procesos de formación y acompañamiento como una forma de empoderar a los pueblos indígenas en la defensa y reivindicación de sus derechos, se recomienda retomar esta experiencia para consolidar procesos de desarrollo comunitario.

El monográfico finaliza con la sección de reflexiones educativas de Flores (2018) sobre la investigación e innovación desde los Centros de Apoyo y Desarrollo Educativo Profesional una perspectiva intercultural, la reflexión describe que los Centros de Apoyo y Desarrollo Educativo Profesional promueven la investigación e innovación con una perspectiva intercultural. Es decir, tratan de fomentar la investigación e innovación didáctica, pedagógica y en la generación de nuevas tecnologías para el aprendizaje a partir de un modelo de investigación propia que reconoce la diversidad, multiculturalidad y multilenguaje de las poblaciones enfocada en las soluciones en y para la comunidad. Además, los Centros de Apoyo y Desarrollo Educativo Profesional se caracterizan por la cooperación intra e interinstitucional en la transferencia de buenas prácticas que apoyan, cultivan, adaptan, comunican, innovan y acogen a la comunidad universitaria.

En definitiva, el lector encontrará en este número distintas perspectivas que abordan Investigaciones e Innovaciones Educativas, así como reflexiones educativas que nos instan a que abordemos. Queda mucho camino por recorrer sobre la investigación y experiencias educativas innovadoras en los procesos de aprendizaje en el contexto nacional e internacional para mejorar la calidad de aprendizaje, en la reproducción del conocimiento por los estudiantes, hacia una docencia motivadora que también pueda integrar el ecosistema digital.

\section{Lista de referencia}

Alemán-Gudiel, P., Alemán-Gudiel, E., \& López, E. (2017). Aprendizaje de las fracciones en estudiantes de séptimo grado del instituto San Ramón en Río San Juan de Nicaragua. Ciencia e Interculturalidad, 21(2), 7-15. DOI: http://dx.doi. org/10.5377/rci.v21i2.5598

Aragón-Arguello, A., \& López-Mairena, E. (2018). Enseñanza y aprendizaje del área y perímetro de polígonos regulares: una propuesta didáctica y evaluación en 
educación primaria. Revista Electrónica de Conocimientos, Saberes y Prácicas, 1(2). DOI: https://doi.org/10.30698/recsp.v1i2.11

Banco Mundial. (2018). Desarrollo mundial "aprender para hacer realidad la promesa sobre la educación". Washington: The World Bank Group

Flores-Morales, J., \& Zamora-Díaz, W. (2016). Unidades didácticas: por una enseñanza asistida de la matemática, Revista Universitaria del Caribe, 16(1), 7-12. DOI: http://dx.doi.org/10.5377/ruc.v16i1.3225

Flores, W. O., \& Gutiérrez y Restrepo, E., León, O., Sarraipa, J., Pantoja, C., Merino, C., Calderón, D., Guinocchio, M., Rivera, M., Calderón, M., E., \& Boticario, J., G. (2016). Centros de Apoyo y Desarrollo Educativo Profesional para la observación y disminución de la deserción universitaria. Ciencia e Interculturalidad, 18(1), 48-62. DOI: http://dx.doi.org/10.5377/rci.v18i1.3049

Flores, W., O. (2018). Evaluación de los aprendizajes en la educación intercultural. Revista Electrónica de Conocimientos, Saberes y Prácticas, 1(2). DOI: https://doi. org/10.30698/recsp.v1i2.12

Flores, W., O. (2018). Investigación e Innovación desde los Centros de Apoyo y Desarrollo Educativo Profesional una perspectiva intercultural. Revista Electrónica de Conocimientos, Saberes y Prácticas, 1(2). DOI: https://doi.org/10.30698/recsp. v1i2.15

Flores, W., O., \& Auzmendi, E. (2018). Actitudes hacia las matemáticas y su relación con las variables género y etnia. Profesorado. Revista de Currículo y formación del profesorado, 22(3), 231-251.

Flores, W., O., \& López-Mairena, C. (2018). Investigación y experiencia educativas innovadoras en los procesos de aprendizaje. Revista Electrónica de Conocimientos, Saberes y Prácticas, 1(2). DOI: https://doi.org/10.30698/recsp.v1i2.8

Flores, W., O., \& López, E., C. (2016). Recursos didácticos y tecnológicos para la enseñanza de la integral definida en el modelo de Universidad Comunitaria Intercultural. Ciencia e Interculturalidad, 18(1), 63-78. DOI: http://dx.doi. org/10.5377/rci.v18i1.3050

Flores, W., O., \& Olivar-Molina, S. (2016). Actitudes hacia la estadística en la formación del profesorado para contextos multiculturales. Revista Universitaria del Caribe, 18(1), 27-37. DOI: http://dx.doi.org/10.5377/ruc.v17i2.3235 
García-Soto, Y., Flores, W. O., \& Olivar-Molina, A. (2018). Competencias emocionales en la formación del profesorado de educación secundaria y su relación con las actitudes hacia las matemáticas. Revista Electrónica de Conocimientos, Saberes y Prácticas, 1(2). DOI: https://doi.org/10.30698/recsp.v1i2.9

IEEPP. (2016). La eficacia escolar y calidad educativa en Nicaragua. Managua: IEEPP.

IESALC. (2008). Tendencias de la Educación Superior en América Latina y el Caribe. Francia: UNESCO.

Jiménez-Bonilla, E., \& Flores, W., O. (2017). Actitudes hacia las matemáticas: un estudio en una escuela rural de la Costa Caribe Sur de Nicaragua, Revista Universitaria del Caribe, 18(1), 7-16. DOI: http://dx.doi.org/10.5377/ruc.v17i2.3235

López-Mairena, E., \& Calero-Borge, W. (2018). Acompañamiento en procesos de formación a jóvenes líderes de los pueblos indígenas del Pacífico Centro-Norte de Nicaragua. Revista Electrónica de Conocimientos, Saberes y Prácticas, 1(2). DOI: https://doi.org/10.30698/recsp.v1i2.14

Orozco-Alvarado, J., \& Díaz-Pérez, A. (2018). ¿Cómo redactar los antecedentes en una investigación cualitativa?. Revista Electrónica de Conocimientos, Saberes y Prácticas, 1(2). DOI: https://doi.org/10.30698/recsp.v1i2.13

Pérez-Obregón, J., \& Romero-Díaz, T. (2018). Análisis del rendimiento académico mediante regresión logística y múltiple. Revista Electrónica de Conocimientos, Saberes y Prácicas, 1(2). DOI: https://doi.org/10.30698/recsp.v1i2.10

Romero-Díaz, T., \& Guzmán-Contreras, J. (2016). Evaluación a profesores en las competencias matemáticas de Educación Media, Juigalpa, 2014. Ciencia e Interculturalidad, 18(1), 22-32 DOI: http://dx.doi.org/10.5377/rci.v18i1.3047

Suárez-Martínez, M., Martínez-Ortiz, J., \& López-Mairena, E, (2017). Aprendizaje de la circunferencia aplicando el Modelo de Van Hiele en estudiantes de undécimo grado de Educación Secundaria de Río San Juan. Revista Universitaria del Caribe, 18(1), 17-22. DOI: http://dx.doi.org/10.5377/ruc.v18i1.4796 